\title{
Real Time Hybrid Simulation with Online Model Updating: An Analysis of Accuracy
}

\author{
$\mathrm{Ge} \mathrm{Ou}^{a, 1}$, Shirley J. Dyke ${ }^{a, b}$, Arun Prakash ${ }^{a}$ \\ ${ }^{a}$ School of Civil Engineering, Purdue University, West Lafayette, IN 47906, USA. \\ ${ }^{b}$ School of Mechanical Engineering, Purdue University, West Lafayette, IN 47906, USA.
}

\begin{abstract}
In conventional hybrid simulation (HS) and real time hybrid simulation (RTHS) applications, the information exchanged between the experimental substructure and numerical substructure is typically restricted to the interface boundary conditions (force, displacement, acceleration, etc.). With additional demands being placed on RTHS and recent advances in recursive system identification techniques, an opportunity arises to improve the fidelity by extracting information from the experimental substructure. Online model updating algorithms enable the numerical model of components (herein named the target model), that are similar to the physical specimen to be modified accordingly. This manuscript demonstrates the power of integrating a model updating algorithm into RTHS (RTHSMU) and explores the possible challenges of this approach through a practical simulation. Two Bouc-Wen models with varying levels of complexity are used as target models to validate the concept and evaluate the performance of this approach. The constrained unscented Kalman filter (CUKF) is selected for using in the model updating algorithm. The accuracy of RTHSMU is evaluated through an estimation output error indicator, a model updating output error indicator, and a system identification error indicator. The results illustrate that, under applicable constraints, by integrating model updating into RTHS, the global response accuracy can be improved when the target model is unknown. A discussion on model updating parameter sensitivity to updating accuracy is also presented to provide guidance for potential users.
\end{abstract}

Keywords: Constrained Unscented Kalman Filter, Model Updating, Nonlinear

${ }^{1}$ Corresponding Author, Email: gou@ prudue.edu

Preprint submitted to Journal of $\mathrm{BT}_{E} X$ Templates

May 6, 2016

(C) 2016. This manuscript version is made available under the Elsevier user license http://www.elsevier.com/open-access/userlicense/1.0/ 
System Identification, Real Time, Hybrid Simulation

\section{Introduction}

In contrast to conventional testing methods and pure numerical simulation, in hybrid simulation (also known as pseudo-dynamic testing or dynamic sub-structuring) the structural system is decomposed into two substructures, namely the numerical substructure (which is usually well understood) and the experimental substructure (whose behavior is to be studied). For simulation, the two substructures of HS are linked by a transfer system (hydraulic actuator, shake table, electric motors etc.) to enforce continuity of the solution at the interface. Both the local performance (specimen level) and the global performance (structural level) can be observed and evaluated, and thus HS is considered to be a cost/space/time efficient approach compared to traditional shake table testing [41]; [29].

For several decades, HS were exclusively implemented using extended time, thus neglecting any rate-dependent effects associated with structural components [26], [42], [34]. Recent advances have encouraged the development of HS methods that are executed at a real time scale, named real time hybrid simulation (RTHS) [7], [8], [16].

One tempting benefit of RTHS is that only the critical component must be fabricated and tested, the remainder of the structure can be numerically modeled. Therefore it is intuitive to choose auxiliary devices (MR dampers, base isolation, etc.) as the experimental substructure, because the functionality of those devices (normally for vibration control purposes) is unique and quite distinguishable from the structural components. However, for evaluating structures in which a given component (column, bridge pier, structural connection) may be used in multiple instances in the structure, one might take the approach of using a limited number of the repeated components as the critical physical specimens and leave the rest in the simulation. Therefore, the modeling error of these similar components may contribute significantly to the global response, and affect the fidelity of a RTHS. Thus, estimation of the numerical model of those nonlinear components during HS can preserve the fidelity of the experiment.

This opportunity has recently been recognized by researchers developing novel 
testing approaches. Rather than only exchanging information at the interface (displacement, acceleration or restoring force), information to improve the numerical substructure can also be extracted from the response of the experimental substructure. It can then be used to improve the representation of similar components in the numerical model. Kwon et al. [24] first introduced the concept of representing an entire structure with several key physical components, and modifying their numerical models using the physical response in real-time. The numerical model used in simulation consisted of a collection of Bouc-Wen models with predetermined parameters. During HS, a weighting factor was identified for each Bouc-Wen model until the summation of their weighted responses matched the measured physical response. Thus, the accuracy of this approach highly depends on the chosen initial collection of Bouc-Wen models. In

40 the subsequent two years, several techniques to apply model updating in HS have been developed, mostly in the unscented Kalman filter family. Those approaches include using the constrained unscented Kalman filter (CUKF) in RTHS [46], [49] and the unscented Kalman filter (UKF) algorithm in HS [18] and RTHS [28] to identify BoucWen model parameters. Experimental results in the aforementioned work demonstrate the feasibility of model updating in HS and the associated improvement in testing accuracy.

Although HS with model updating (HSMU) has been experimentally validated, an evaluation of the limitations of HSMU has not been performed. For both HSMU and RTHSMU, online model updating algorithms require knowledge of the excitation to the experimental substructure as well as its response to identify the model parameters. This excitation normally takes the form of a structural response which is already filtered (the structure itself is a filter) and likely contains limited frequency information, especially on examining the dynamic system where specimen response is rate dependent. In HS, the identification information is more related to amplitude where the loading does not contain frequency content with low speed execution. While in RTHS, the information maybe related to both amplitude and frequency. For system identification, this filtered excitation may be insufficient or provide limited useful information. Other possible limitations relate to the varying level of complexity of the nonlinear models to be identified. In addition, parameter convergence in model updating may affect the 


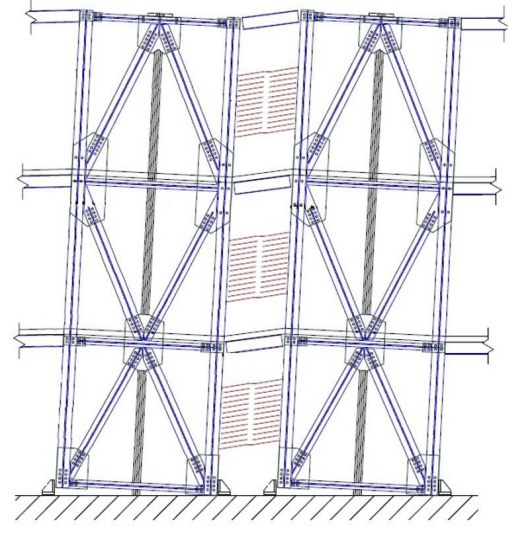

(a) Rocking Frame with Connection Plates

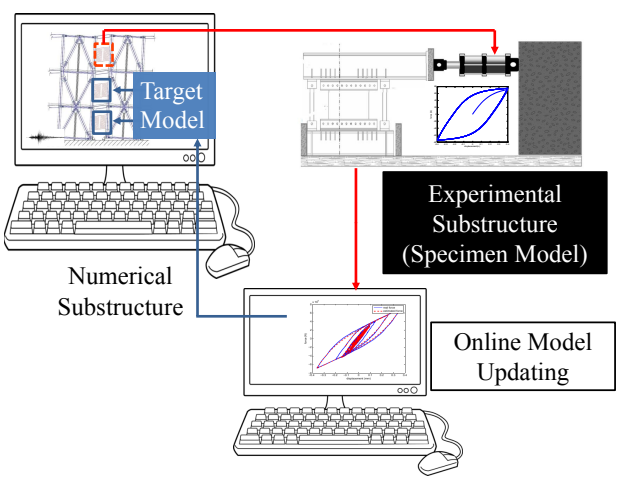

(b) Concept of HSMU

Figure 1: Simulation Case Study

which in this case is simply the upper shear plate. The nonlinear behavior of the specimen model is represented using Bouc-Wen models with different levels of complexity. The other two plates with the same nominal design are included within the numerical substructure, each defined to be a target model. The initial parameters of the target models are unknown (selected parameters are far away from the specimen model) and are expected to be updated using information about the specimen model acquired during the RTHS. The CUKF is used as the online nonlinear model identification method. This RTHSMU simulation (Fig. 1(b)) includes: the numerical substructure consisting 
of frames with PT strands, and target model which is updated with real-time parameters; an experimental substructure which only consists of the specimen model; and the online model updating algorithm. The performance of RTHSMU simulation is first presented at the global level, where the self-centering frame behavior is compared to baseline simulation results without any modeling errors in either the target model or the specimen model, and then discussed thoroughly on the component level in terms of the model updating. The evaluation and validation of the model updating performance considers the following: richness of the input (broadband signal, structural response, sinusoidal signal, etc.), complexity of the target model, and selected tunable variables in the model updating algorithm.

\section{Real Time Hybrid Simulation with Model Updating}

To incorporate model updating into the RTHS formulation some modifications to the RTHS procedure are needed. To demonstrate the modified procedure, Bouc-Wen (BW) elements are used to represent typical component nonlinearities. The CUKF algorithm is used herein to identify the BW parameters in real-time using the formulation provided. The entire study has been verified in terms of real time execution, however, in this study, the simulation is not running on a RT platform.

Consider the equation of motion for a reference structural with nonlinear components in conventional simulation written as:

$$
M \ddot{x}+C \dot{x}+K x+R\left(x, \dot{x}, \theta_{R}\right)=-M \Gamma \ddot{x}_{g}
$$

where $\mathrm{M}, \mathrm{C}, \mathrm{K}$ are the linear mass, damping, stiffness matrices of the reference structure, $\left.R\left(x, \dot{x}, \theta_{R}\right)\right)$ is the restoring force provided by the nonlinear components, $\theta_{R}$ are the parameters of the nonlinear components, $x, \dot{x}$ and $\ddot{x}$ are structural responses (displacement, velocity, acceleration), and $\ddot{x}_{g}$ denotes earthquake excitation.

We write the equations of motion for HS with model updating in the form:

$$
M^{N} \ddot{x}^{N}+C^{N} \dot{x}^{N}+K^{N} x^{N}+F^{E}\left(x^{E}, \dot{x}^{E}\right)+R^{N}\left(x^{N}, \dot{x}^{N}, \tilde{\theta}_{R}\right)=-M \Gamma \ddot{x}_{g}
$$




$$
\begin{gathered}
M^{E} \ddot{x}^{E}+C^{E} \dot{x}^{E}+K^{E} x^{E}+R^{E}\left(x^{E}, \dot{x}^{E}\right)=F^{E}\left(x^{E}, \dot{x}^{E}\right) \\
\tilde{\theta}_{R}=\Psi\left(R^{E}, x^{E}, \dot{x}^{E}, \theta_{\Psi}\right)
\end{gathered}
$$

where the superscript ( $)^{N}$ and ()$^{E}$ denote the portions of the reference structure included in the numerical and experimental substructures respectively, $M=M^{E}+M^{N}$, $C=C^{E}+C^{N}, K=K^{E}+K^{N} . F^{E}$ denotes the measured force in the experimental substructure. $\Psi$ indicates the model updating is performed in real-time, $\theta_{\Psi}$ is the parameter being updated through the chosen model updating algorithm and $\tilde{\theta}_{R}$ is the recursively identified nonlinear model parameter that minimizes the associated cost function. Thus, in this implementation, the numerical restoring force $R^{N}\left(x^{N}, \dot{x}^{N}, \tilde{\theta}_{R}\right)$ adapts in real-time based on the physical responses.

\subsection{Modeling of Hysteresis}

The presence of nonlinear behavior is most prominent in the restoring force and is often desirable for energy dissipation under extreme loading. Physical material models are often either too simple and insufficient to accurately capture the hysteresis (e.g. bilinear model, elastic-perfect plastic model) or too complex to be used in practical applications in RTHS (Menegotto-Pinto model) [27]. Instead, a black box model combined with proper physical understanding of the component hysteretic behavior is an ideal trade-off [20]. Such a hysteretic phenomenological model was first proposed by Bouc [5] and then modified by Wen [48]. Ever since, variations on the Bouc-Wen model have been introduced to capture more complex material hysteresis such as pinching and degrading [3], [6], [30]. Those models have been validated experimentally and shown to be applicable to represent inelastic steel nonlinearity several decades [25], [39].

Two Bouc-Wen models are considered in this case study, including the classic Bouc-Wen model (denoted here as the General BW, or GBW in the manuscript) model stated in Eq. 5 and 6.

$$
R_{B 1}\left(x^{E}, z\right)=\alpha_{B 1} k_{B 1} x^{E}+(1-\alpha) k_{B 1} z
$$




$$
\dot{z}=A_{B 1} \dot{x}^{E}-\beta_{B 1}\left|\dot{x}^{E}\right||z|^{n_{B 1}-1} z-\gamma_{B 1} \dot{x}^{E}|z|^{n_{B 1}}
$$

where $k_{B 1}$ is the stiffness coefficient and $0 \leq \alpha_{B 1} \leq 1$ determines the level of nonlinearity, $\alpha_{B 1}=1$ indicates the system is purely linear and $\alpha_{B}=1$ indicates the system is purely hysteretic. $A_{B 1}, \beta_{B 1}, n_{B 1}, \gamma_{B 1}$ governs the shape of the hysteresis loop.

In order to capture a greater variety of material behavior, an extended Bouc-WenBaber-Noori model [4], [3] model (denoted here as the Extended BW, or EBW) is considered, this model includes pinching and degradation effects of a structure component, represented in Eq. 7 - 15.

$$
R_{B 2}\left(x^{E}, z\right)=\alpha_{B 2} k_{B 2} x^{E}+(1-\alpha) k_{B 2} z
$$

$\alpha_{B 2}$ and $k_{B 2}$ has similar definition as $\alpha_{B 1}$ and $k_{B 1}$ in the GBW.

$$
\dot{z}=h(z)\left\{\frac{\dot{x}^{E}-\nu(\varepsilon)\left(\beta_{B 2}\left|\dot{x}^{E}\right||z|^{n_{B 2}-1} z+\gamma_{B 2} \dot{x}^{E}|z|^{n_{B 2}}\right.}{\eta(\varepsilon)}\right\}
$$

and, response duration and severity is measured by $\varepsilon(t)$ which is proportional to energy dissipation $E(t)$ in Eq. 9.

$$
\begin{gathered}
E(t)=\int\left(1-\alpha_{B 2}\right) k_{B 2} z \dot{x}^{E} d t, \varepsilon(t)=\int z \dot{x}^{E} d t \\
\nu(\varepsilon)=1+\delta_{\nu B 2} \varepsilon \\
\eta(\varepsilon)=1+\delta_{\eta B 2} \varepsilon
\end{gathered}
$$

where $\nu$ and $\eta$ are degradation shape function, and $\delta_{\nu B 2}, \delta_{\eta B 2}$ are degradation parameters. $h(z)$ is the pinching function given by Eq. 12-15.

$$
\begin{gathered}
h(z)=1-\zeta_{1 B 2} e^{-\left[z \cdot \operatorname{sgn}\left(\dot{x}^{E}\right)-q_{B 2} z_{x} E\right]^{2} / \zeta_{2 B 2}^{2}} \\
\zeta_{1}(\varepsilon)=\zeta_{s B 2}\left(1-e^{-p_{B 2} \varepsilon}\right) \\
\zeta_{2}(\varepsilon)=\left(\Psi_{B 2}+\delta_{\Psi B 2} \varepsilon\right)\left(\lambda_{B 2}+\zeta_{1}\right)
\end{gathered}
$$




$$
z_{x^{E}}=\left[\frac{1}{\nu(\varepsilon)\left(\beta_{B 2}+\gamma_{B 2}\right)}\right]^{\frac{1}{n_{B 2}}}
$$

The parameters $\zeta_{s B 2}, p_{B 2}, q_{B 2}, \Psi_{B 2}$ and $\delta_{\Psi B 2}$ are involved in the pinching function. Thus, the complete parameters set for the EBW is $\alpha_{B 2}, k_{B 2}, \beta_{B 2}, n_{B 2}, \delta_{\eta B 2}, \delta_{\nu B 2}$, $q_{B 2}, \gamma_{B 2}, \zeta_{s B 2}, p_{B 2}, \Psi_{B 2}, \delta_{\Psi B 2}, \lambda_{B 2}$.

The accuracy of the system identification will depend on the sensitivity of the system response to each parameter in the model. It would be difficult to obtain a good estimate of the global solution if each of the model parameters do not participate in the response. Such an analysis is normally implemented numerically based on the variation of a given parameter set (base value) to determine the quantitative change in the output. This is known as a local sensitivity analysis when each parameter is considered in isolation. When all of the parameters vary simultaneously in a given range, and the ranking of each is evaluated, it is referred to as a global sensitivity analysis. Sobol indices [38] are used for evaluate parameter global sensitivity, as suggested in [25]. $S_{j}$ in the Sobol indices indicates the effect of each individual parameter. Because the analysis is performed numerically, the result (sensitivity ranking) depends on the choice of the base value set. The base value sets chosen in this analysis are determined using the finite element model of the shear plate, model behaviors are demonstrated in Fig. 2(b) and 2(d). The result of sensitivity each parameter in the GBW and EBW is presented in Tables 1 and 2.

\begin{tabular}{cc|c|cc}
\multicolumn{5}{c}{ Table 1: General Bouc-Wen Model Sensitivity Ranking } \\
\hline Parameter & Base Value & Local Ranking & $S_{i}$ & Global Ranking \\
\hline$\alpha_{B 1}$ & 0.35 & 5 & $7.96 \%$ & 5 \\
$k_{B 1}$ & 35 & 2 & $31.8 \%$ & 1 \\
$A_{B 1}$ & 15 & 3 & $10.39 \%$ & 4 \\
$\beta_{B 1}$ & 1.7 & 4 & $18.04 \%$ & 3 \\
$n_{B 1}$ & 1.7 & 1 & $28.31 \%$ & 2 \\
$\gamma_{B 1}$ & 0.5 & 6 & $2.45 \%$ & 6 \\
\hline
\end{tabular}

The sensitivity analysis of the GBW indicates that each parameter contributed more 
Table 2: Extended Bouc-Wen Model Sensitivity Ranking

\begin{tabular}{cc|c|cc}
\hline Parameter & Base Value & Local Ranking & $S_{i}$ & Global Ranking \\
\hline$\alpha_{B 2}$ & 0.03 & 4 & 0.0941 & 4 \\
$k_{B 2}$ & 291.67 & 2 & 0.39 & 1 \\
$\beta_{B 2}$ & 4.98 & 3 & 0.14 & 3 \\
$n_{B 2}$ & 1.41 & 1 & 0.18 & 2 \\
$\delta_{\eta B 2}$ & 0.33 & 6 & 0.0283 & 7 \\
$\delta_{\nu B 2}$ & 0.12 & 5 & 0.0421 & 5 \\
$q_{B 2}$ & 0.011 & 9 & 0.0139 & 10 \\
$\gamma_{B 2}$ & 0.089 & 7 & 0.0024 & 12 \\
$\zeta_{s B 2}$ & 0.13 & 8 & 0.0212 & 9 \\
$p_{B 2}$ & 0.023 & 10 & 0.0226 & 8 \\
$\Psi_{B 2}$ & 0.64 & 11 & 0.0031 & 11 \\
$\delta_{\Psi B 2}$ & 0.016 & 12 & 0.0313 & 6 \\
$\lambda_{B 2}$ & 0.18 & 13 & 0.001 & 13 \\
\hline
\end{tabular}
works for linear systems, the extended Kalman Filter (EKF) [23] and the Unscented Kalman Filter (UKF) [21] were developed subsequently for nonlinear systems. With the EKF the estimation is optimized based on linear approximations of the nonlinear 
system through a Jacobian matrix, while UKF uses the unscented transformation (UT). A large amount of effort has been dedicated to comparing the performance of EKF and UKF, with conclusions that vary based on the degree of nonlinearity in the selected model and the application of interest [32], [33], [9], [15], [47]. One significant benefit of using UKF is that no Jacobian matrix is needed. The UKF is extended from estimating the state vector to parameter identification, and has been applied to estimate Bouc-Wen model parameter sets [50] through numerical validation. The results indicate that the UKF can be used effectively for nonlinear structural identification, and further extensions consider experimental validation in [40]. This approach continues to be recognized as being effective for online HS applications [18], [28].

In the Bouc-Wen model series, certain model parameters have physical meaning, such as $\alpha_{B, i}$ which determines the severity of structure nonlinearity and has a range limit of $0 \leq \alpha_{B, i} \leq 1, k_{B, i}$ is the initial linear stiffness of the component which should be non-negative. Thus, the nonlinear system identification problem becomes a parameter estimation with interval constraints. The CUKF algorithm has been developed for state estimation with interval constraints, and has been experimentally validated in structural testing applications involving the Bouc-Wen model in RTHS [46], turbofan engines states estimation [35], and tire force, velocity etc. estimation for vehicles [31].

Consider a stochastic nonlinear discrete-time dynamic system:

$$
\begin{aligned}
\theta_{k} & =F\left(\theta_{k-1}, u_{k-1}, k-1\right)+w_{k-1} \\
y_{k} & =H\left(\theta_{k}, k\right)+v_{k}
\end{aligned}
$$

where $F$ and $H$ are process and observation models. For a parameter estimation problem, $\theta_{k-1}$ is the system parameter vector. Assume for all $k \geq 1$, input $u_{k}$, measurement $y_{k}$, and the PDFs of $\rho\left(\theta_{0}\right), \rho(w), \rho(v)$ are known. Also, $w$ and $v$ are the process noise and measurement noise, with zero mean and known variances, represented by $Q$ and $R . \theta_{0}$ is the initial condition (guess) of the parameter estimation vector.

Unlike the UKF, CUKF projects the sigma points $\Theta_{k}$ with interval constraints named the interval constraint unscented transformation (ICUT), assuming the given interval constraints $d_{k} \leq \theta_{k} \leq e_{k}$. The ICUT procedure is defined as $\left[\gamma_{k}, \Theta_{k}\right]=$ 190

$\Phi_{I C U T}\left(\hat{\theta}_{k}, P_{k}^{\theta \theta}, d_{k}, e_{k}, L, \lambda_{C}\right)$, where $\gamma_{k}$ is the weighted factor associated with each 
sigma point, $\hat{\theta}_{k}$ is the estimated mean at each step, $L$ is the number of parameter to be identified, $\lambda_{C}$ is predefined ICUT parameter $\lambda_{C}>-L$ [43].

Once the ICUT is defined, the forecast step is given as:

$$
\begin{aligned}
& {\left[\gamma_{k-1}, \Theta_{k-1 \mid k-1}\right]=\Phi_{I C U T}\left(\hat{\theta}_{k}, P_{k}^{\theta \theta}, d_{k}, e_{k}, L, \lambda_{C}\right)} \\
& \Theta_{j, k \mid k-1}=F\left(\Theta_{k-1 \mid k-1}, u_{k-1}, k-1\right)+w_{k-1} \\
& \hat{\theta}_{k \mid k-1}=\sum_{j=0}^{2 L} \gamma_{j, k-1} \Theta_{j, k \mid k-1} \\
& P_{k \mid k-1}^{\theta \theta}=\sum_{j=0}^{2 L} \gamma_{j, k-1}\left[\Theta_{j, k \mid k-1}-\hat{\theta}_{k \mid k-1}\right]\left[\Theta_{j, k \mid k-1}-\hat{\theta}_{k \mid k-1}\right]^{T}+Q_{k-1} \\
& {\left[\gamma_{k}, \Theta_{k \mid k-1}\right]=\Phi_{I C U T}\left(\hat{\theta}_{k \mid k-1}, P_{k \mid k-1}^{\theta \theta}, d_{k}, e_{k}, L, \lambda_{C}\right)} \\
& \mathcal{Y}_{j, k \mid k-1}=H\left(\Theta_{j, k \mid k-1}, k\right) \\
& \hat{y}_{k \mid k-1}=\sum_{j=0}^{2 L} \gamma_{j, k} \mathcal{Y}_{j, k \mid k-1} \\
& P_{k \mid k-1}^{y y}=\sum_{j=0}^{2 L} \gamma_{j, k}\left[\mathcal{Y}_{j, k \mid k-1}-\hat{y}_{k \mid k-1}\right]\left[\mathcal{Y}_{j, k \mid k-1}-\hat{y}_{k \mid k-1}\right]^{T}+R_{k} \\
& P_{k \mid k-1}^{\theta y}=\sum_{j=0}^{2 L} \gamma_{j, k}\left[\Theta_{j, k \mid k-1}-\hat{\theta}_{k \mid k-1}\right]\left[\mathcal{Y}_{j, k \mid k-1}-\hat{y}_{k \mid k-1}\right]^{T}
\end{aligned}
$$

$P_{k \mid k-1}^{\theta \theta}$ is the forecast error covariance, $P_{k \mid k-1}^{y y}$ is the innovation covariance, $P_{k \mid k-1}^{\theta y}$ is the cross covariance. Similar to classic KF update, the estimate step (also known as data assimilation step) is defined:

$$
\begin{aligned}
& K_{k}=P_{k \mid k-1}^{\theta y}\left(P_{k \mid k-1}^{y y}\right)^{-1} \\
& \hat{\theta}_{k \mid k}=K_{k}\left(y_{k}-\hat{y}_{k \mid k-1}\right) \\
& P_{k \mid k}^{\theta \theta}=P_{k \mid k-1}^{\theta \theta}-K_{k} P_{k \mid k-1}^{y y} K_{k}^{T}
\end{aligned}
$$

where $K^{k}$ is the Kalman gain matrix and $P_{k \mid k}^{\theta \theta}$ is the data-assimilation error-covariance.

In summary, to apply the CUKF user-defined parameters include the initial guess of the parameter vector $\theta_{0}$, the ICUT parameter $\lambda_{C}$, the process noise variance $Q$ and measurement noise variance $R$. The choices of those parameters may affect the accuracy of system identification as discussed subsequently. 


\section{Simulation case study}

The numerical example for demonstrating and validating RTHSMU is a controlled,

200 self-centering frame proposed by Deierlein et al, [11], [12], [13], [14] which is composed of two steel frames, vertical post tension strands in both frames, and the two frames are linked by three identical shear connection as shown in Fig. 1(a). The design concept is as follows: 1) the steel frames with post tension strands act as a two stage linear (bilinear) frame determined by the initial force in the strands and the top displacement; 2) the vertical post-tension strands provide the self-centering force; and, 3 ) the replaceable shear connection plates dissipate energy during dynamic loading. It is considered to be a very practical application of RTHS analysis because the bilinear behavior of the frames with PT strands can be isolated in the numerical substructure. Meanwhile, model updating is beneficial since all the connection plates are identical and experience similar loading paths.

To demonstrate the RTHSMU approach, the structure is represented with a bilinear frame model and GBW/EBW models. The simplified model responses are compared to the OpenSees finite element model available within the NEES data repository [10]. The resulting comparison is provided in Fig. 2, and indicates a slight difference in the hysteresis behavior (see Fig. 2(b) and 2(d)). At very large displacements the shear plate will deform out-of-plane and this type of hysteresis is not captured by the BW model family. Here the parameters for both the GBW and EBW models are determined through genetic algorithms by minimizing the error between the BW model response and finite element model response. The nominal values of each parameter are listed in Table 1 and 2. The simplified model with this nominal parameter set are assumed in the sequel as the reference solution in the following analysis which also defines the true parameters for the specimen model.

RTHSMU efficiency is qualitatively demonstrated through a simulation of the case study following the concept described in Fig. 1(b). In this RTHSMU simulation the third story connection plate is assumed to be loaded as the experimental substructure in RTHS, and also modeled using the specimen model parameter (model with nominal parameters) with its input and response available for system identification. The other two 


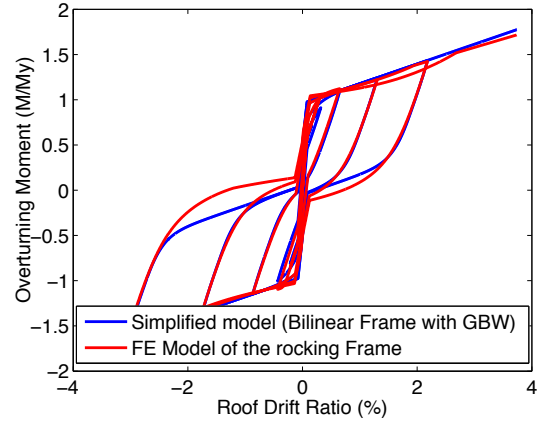

(a) Frame Response using GBW model

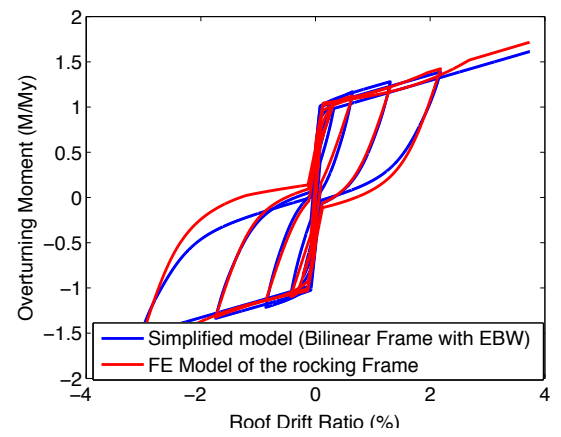

(c) Frame Response using EBW model

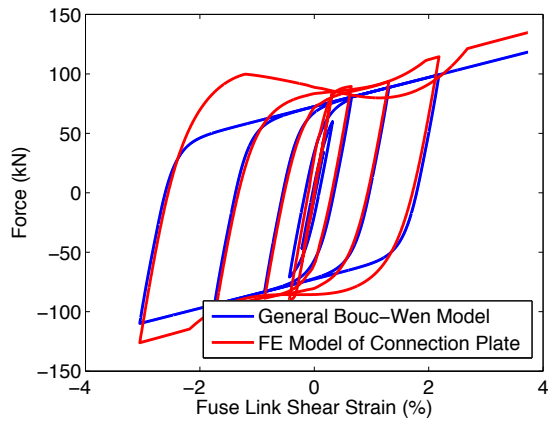

(b) Connection Plate Response using GBW model

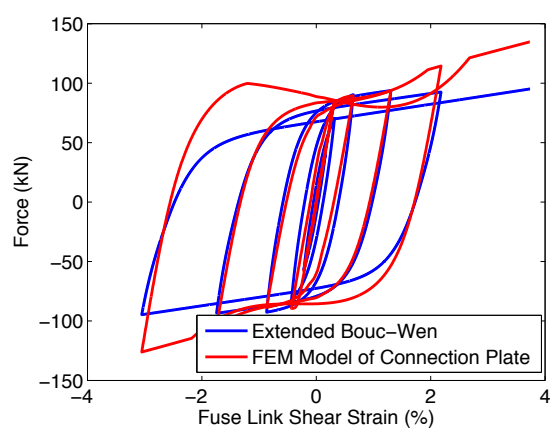

(d) Connection Plate Response using EBW model

Figure 2: Comparison between GBW/EBW Model and FEM Responses 
plates (known as the target models) have initial modeling errors as they are modeled using BW models with 50\% error in each parameters. This model will adopt the estimated parameter sets generated by the model updating algorithm. The RTHSMU results are compared to both a) the baseline simulation result (reference solution) where no modeling error is assumed and b) a simulation representative of conventional RTHS, where only the third story connection plate (specimen model) is accurately loaded and the two remaining plates are modeled numerically with erroneous parameters (50\% error) and do not adopt the updated parameters.

The entire self-centering system (two frames, PT strands and connection plates) is simulated using a scaled El-Centro earthquake excitation, and a comparison of the results is shown in Fig. 3. It is clear that significant error exists in the global response (top displacement-moment of the self-centering frame) using conventional RTHS when there is modeling error in the target model. The RTHSMU response initially exhibits the same conditions as the conventional RTHS. However, after several seconds it converges to the baseline solution as in Fig. 3(b). For the EBW model, the convergence is achieved even faster, inside of 1 second, with the initial model parameters placed $50 \%$ away from the true parameters. This result indicates the attractive benefit offered by RTHSMU which improves the RTHS accuracy without the need for testing all instances of components with modeling uncertainties. This general finding has also been reported in [24], [46], [18], [28]. 


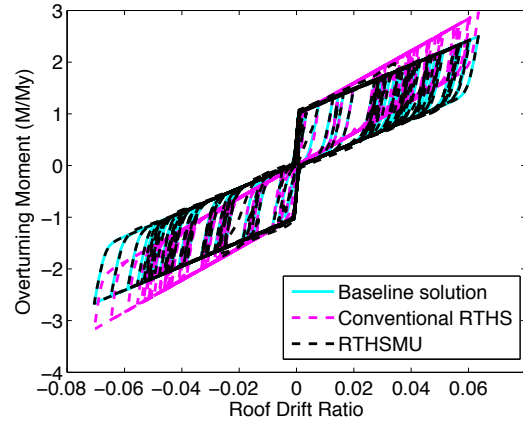

(a) Frame Response Comparison, GBW

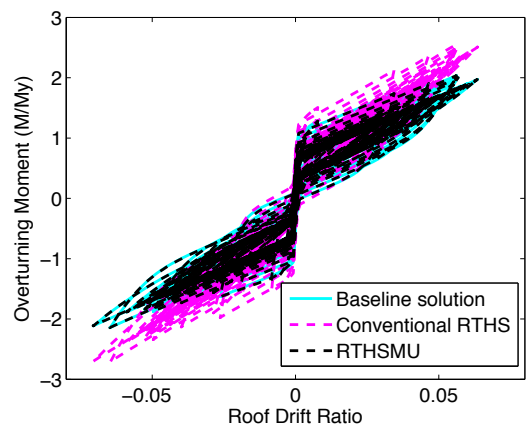

(c) Frame Response Comparison, EBW

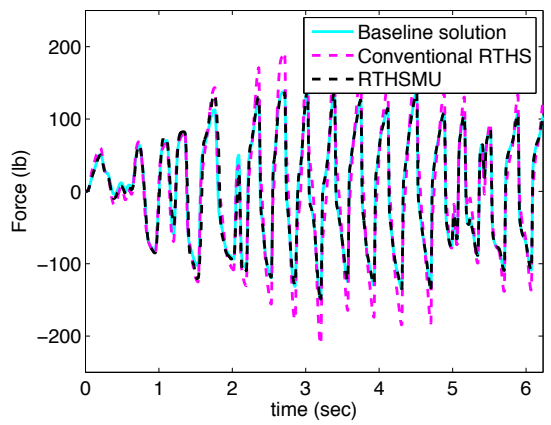

(b) Response from $2^{\text {nd }}$ Story Connection Plate, GBW

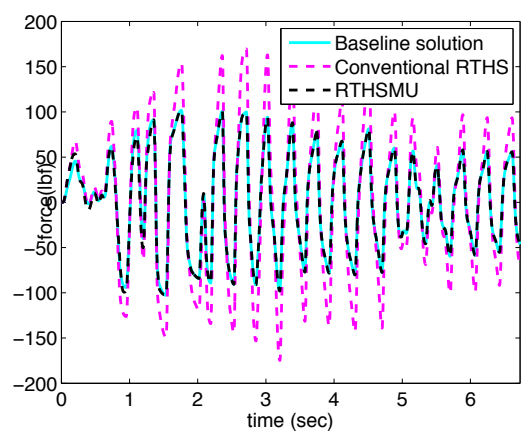

(d) Response from $2^{\text {nd }}$ Story Connection Plate, EBW

Figure 3: Comparison between Baseline Solution, Conventional RTHS and RTHSMU 


\section{Performance of CUKF in RTHS Simulation}

The success of RTHSMU depends highly on the model updating performance. Besides typical factors to be considered in the model updating accuracy analysis, including parameter initial condition $\theta_{0}$, model updating algorithm variable $\left(Q, R\right.$ and $\lambda_{c}$ etc. for CUKF), other concerns specific to RTHSMU should be examined. One limitation of RTHSMU is the incomplete excitation that is important for system identification [2].

\subsection{Excitation persistence analysis}

In RTHSMU, the specimen to be identified is driven by the local structural response which is dominated by a combination of several sinusoidal signals with irregular amplitudes at the structures modes. Depending on the degree of model complexity, this excitation frequency may be limited. Meanwhile, the target model adopts structural parameters in real-time, and also generates the response of that model to a given input.

Thus, it is important to evaluate RTHSMU feasibility through 1) understanding the effect of broadband and narrow-band excitations on identification performance, 2) assessment of the time-varying model output accuracy that adopts the online estimation parameter set, and 3) validating the accuracy of RTHSMU using an alternate excitation. Thus, three levels of accuracy measures are proposed, as shown in Fig. 4: Error indicator 1 (E1) evaluates the estimation output error between CUKF estimated output $\left(Y_{e s t}\right)$ and the reference model output (measurement $Y_{m e s}$ ), and E2 indicates the model updating output error between the time varying model response $\left(Y_{i d}\right)$ and the reference model output $\left(Y_{m} e s\right)$, which is the main indicator to judge the success of RTHSMU. Both E1 and E2 are calculated after model updating algorithm runs 4 sec, assuming the steady state solution achieved. E3 is defined with an alternate input which is sent to A) the specimen model (with true parameter) and B) a new model with identified parameter (after model updating procedure), to compute the error between $V Y_{m e s}$ and $V Y_{i d}$, representing the system identification error.

Three types of input signals are considered in the excitation persistence analysis (Table 3), and their frequency domain information is further demonstrated in Fig. 5. For Case B, the structural response has peaks at $2.88 \mathrm{~Hz}, 8.05 \mathrm{~Hz}$ and $12.3 \mathrm{~Hz}$. 

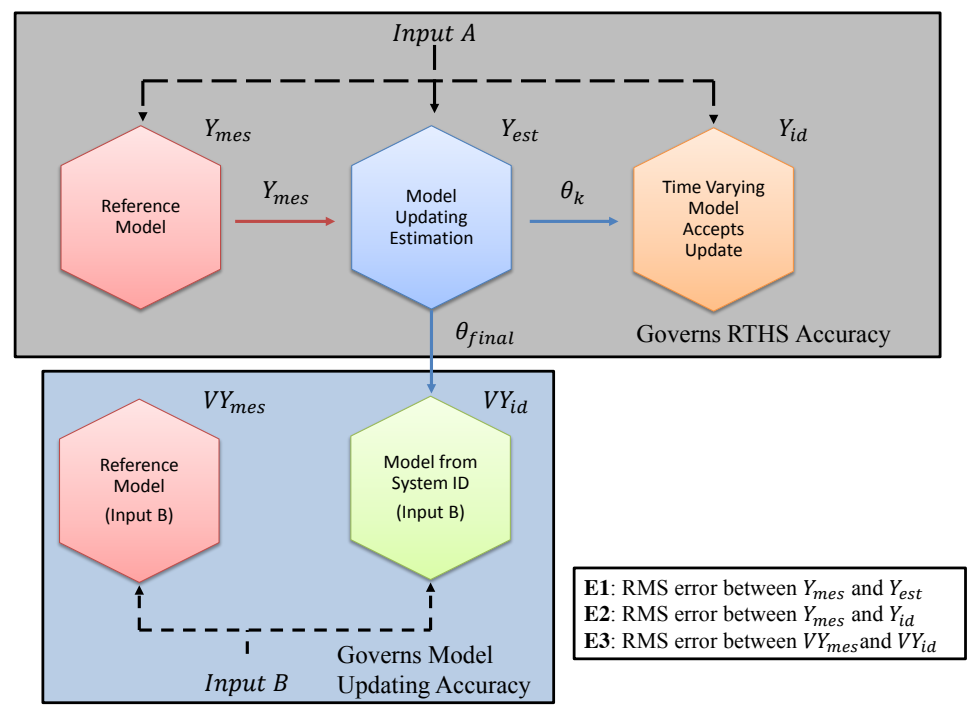

Figure 4: Local Accuracy Measures for RTHSMU

Table 3: Simulation Cases for Excitation Persistence Analysis

\begin{tabular}{c|c|c}
\hline Case No. & Excitation A & Excitation B \\
\hline A & $0-1 \mathrm{~Hz}$ Band limited white noise (BLWN) & Structural Response \\
B & Structural response after earthquake & BLWN \\
C & $0.5 \mathrm{~Hz}$ sine wave with amplitude 3 inch & BLWN \\
D & $0.1,0.5,3 \mathrm{~Hz}$ sine wave with amplitude 1 inch each & BLWN \\
\hline
\end{tabular}

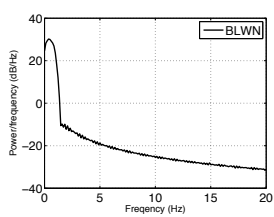

(a) Input-Case $\mathrm{A}$

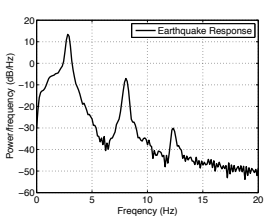

(b) Input-Case B

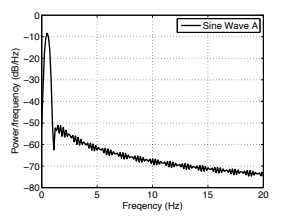

(c) Input-Case $\mathrm{C}$

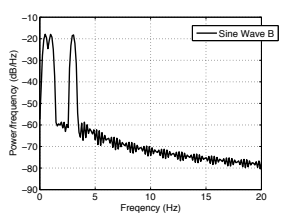

(d) Input-Case D

Figure 5: Excitation signal power spectrum 


\subsubsection{General Bouc-Wen Model}

The nominal (true) parameter for the GBW case is given in Table 1. Because BoucWen model is a phenomenological (mathematical) model, each parameter does not have an explicit physical meaning, the erroneous initial condition used in the target model for model updating has $50 \%$ over-estimation in each value. The results shown in the excitation persistence analysis are computed using the best possible combination of $Q, R, \lambda_{C}$ (see Section 4.2.1. and 4.2.2).

The quantitative RMS error in each case is defined as:

$$
E=\sqrt{\frac{\sum_{i}^{n}\left(R-R_{r e f}\right)^{2}}{n}} / \sqrt{\frac{\sum_{i}^{n}\left(R_{r e f}\right)^{2}}{n}}
$$

\begin{tabular}{c|ccc}
\multicolumn{4}{c}{ Table 4: Error Indices for Excitation Persistence Analysis, GBW } \\
\hline Cases & $E_{1}$ & $E_{2}$ & $E_{3}$ \\
\hline $\mathrm{A}$ & $0.028 \%$ & $0.021 \%$ & $0.25 \%$ \\
$\mathrm{~B}$ & $0.32 \%$ & $0.57 \%$ & $2.0 \%$ \\
$\mathrm{C}$ & $1.23 \%$ & $1.23 \%$ & $\mathbf{9 . 3 3} \%$ \\
$\mathrm{D}$ & $3.57 \%$ & $3.55 \%$ & $2.02 \%$ \\
\hline
\end{tabular}

The model updating results with GBW are shown in Fig. 6 and the quantitative indices are in Table 4. For both case A (BLWN) and B (structural response), all three errors are quite small. It is clear that when the excitation information is broadband and approaching the characteristics of a white noise, the system identification error (E3) is reduced. Thus, the parameter estimation has likely converged to its global optimal solution. However, for a narrow-band excitation (in case C), both E1 and E2 have small value which illustrates the CUKF algorithm is able to estimate a set of model parameters that satisfy the minimum error tolerance, and the time-varying target model using the identified parameter sets $Y_{i d}$ provides an accurate response as compared to $Y_{m e s}$. However, the E3 error is significantly larger using an alternate validation signal (BLWN). With the incomplete excitation, the model updating algorithm generates a local optimal solution specific to the excitation. Thus, it is concluded that not all RTHS cases are suitable for incorporating model updating, and some numerical simulation about model updating behavior in a specific hybrid simulation with a specific 


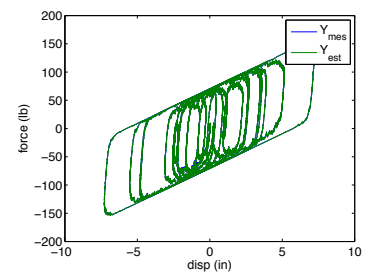

(a) E1-Case A

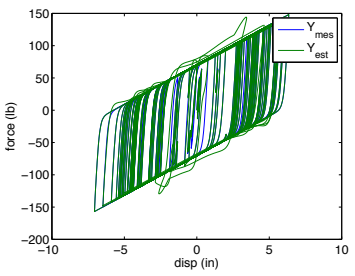

(d) E1-Case B

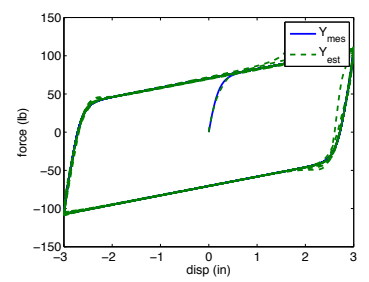

(g) E1-Case C

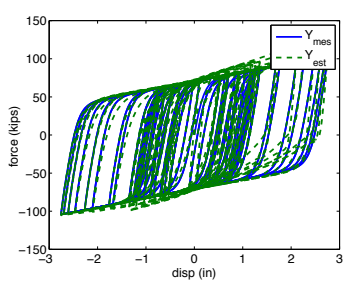

(j) E1-Case D

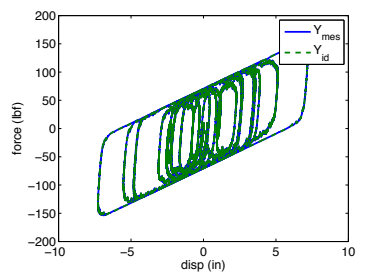

(b) E2-Case A

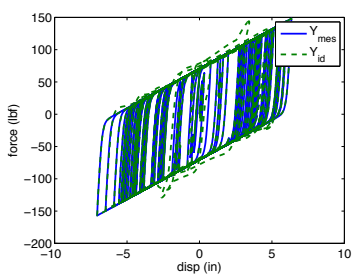

(e) E2-Case B

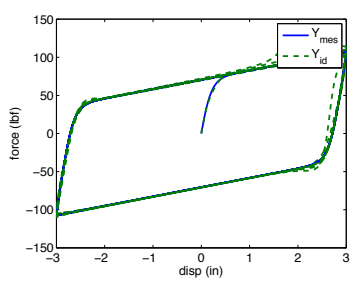

(h) E2-Case C

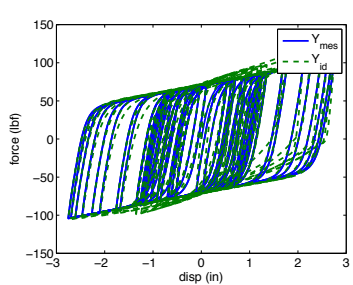

(k) E2-Case D

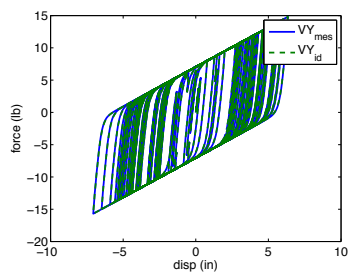

(c) E3-Case A

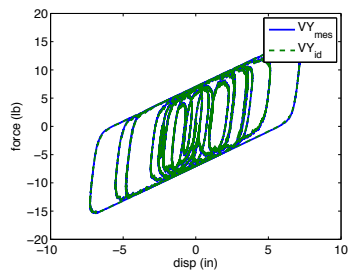

(f) E3-Case B

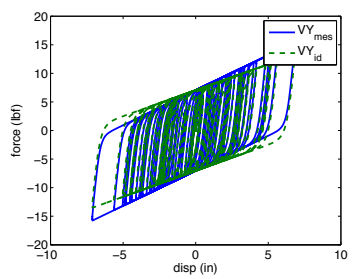

(i) E3-Case C

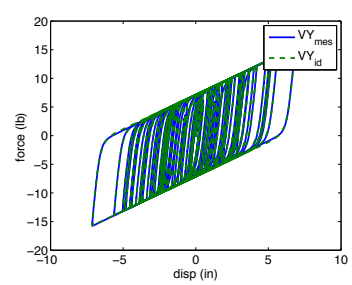

(1) E3-Case D

Figure 6: Real-Time Model Updating Performance and Validation Using Various Excitations, GBW Model 
component model should be examined. This phenomenon is expected to be even more dominant if the model is rate dependent, such as the MR damper.

Fig. 7 shows the parameter convergence. Cases A and B are both assumed to have settled to their global optimal solution indicated by E3, however, only in case A do the parameters reach the nominal values. In case B, although the solution converges after 3 seconds, when the system identification performance is validated through a broader-band signal the final parameter is different from the nominal set. This result may be due to the known fact that the solution of Bouc-Wen model is not unique, as discussed in [19], [20]. Meanwhile, if the excitation signal is not complete, the parameter set is likely to oscillate periodically and not truly converge. This is related to both the excitation signal incompleteness and also the choice of different model updating parameter, $Q, R, \lambda_{c}$, etc. Thus, a model updating parameter study is discussed later.

\subsubsection{Extended Bouc-Wen Model}

A similar study is performed using the EBW model and a 50\% over estimation in each parameter is used. The performance of the model updating procedure for the EBW is shown in Fig. 8, and the quantitative indices are in Table 5. For the EBW model, only the first six parameters which have higher rank in the sensitivity analysis are considered in the parameter convergence analysis in Fig. 9.

\begin{tabular}{c|ccc}
\multicolumn{4}{c}{ Table 5: Error Indices for Excitation Persistence Analysis, EBW } \\
\hline Cases & $E_{1}$ & $E_{2}$ & $E_{3}$ \\
\hline A & $0.27 \%$ & $0.846 \%$ & $5.51 \%$ \\
B & $0.29 \%$ & $0.916 \%$ & $6.64 \%$ \\
C & $0.32 \%$ & $1.63 \%$ & $7.4 \%$ \\
D & $0.48 \%$ & $0.57 \%$ & $2.308 \%$ \\
\hline
\end{tabular}

For the estimated output error index $E_{1}$ and the model updating output error index $E_{2}$, all cases have very good performance indicating that the residual error is very small using the identified parameter sets. However, the system identification error E3 using the alternate validation signal is large as compared to the GBW model for cases A and 


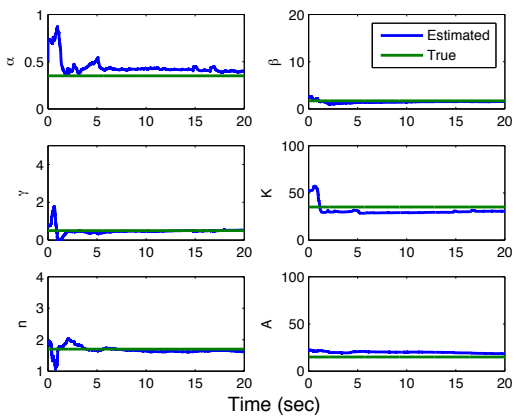

(a) Parameter Convergence - Case A
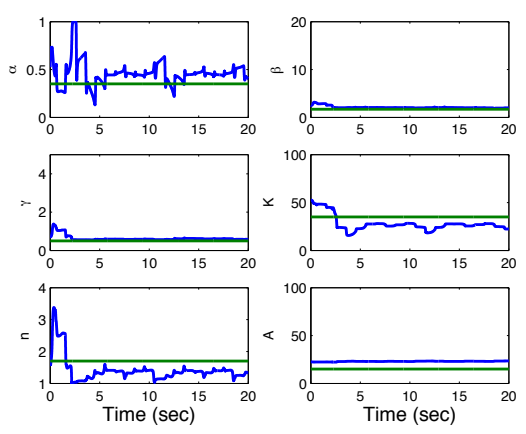

(c) Parameter Convergence - Case C
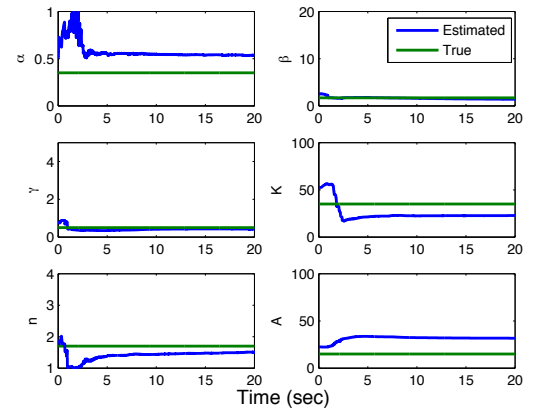

(b) Parameter Convergence - Case B
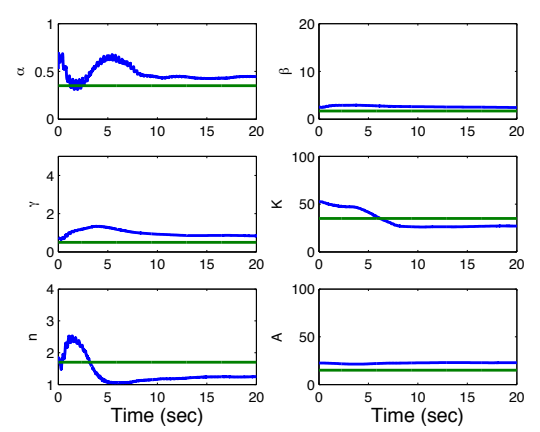

(d) Parameter Convergence - Case D

Figure 7: Parameter Convergence for Excitation Persistence Analysis, General BW model 
B. In this case, the identified parameter sets do not converge to the nominal values even with a BLWN excitation. There are a couple of possible reasons for this. First, the EBW model with the same hysteresis behavior may be represented by several global solutions (i.e. the solution is not unique). Second, based on the sensitivity analysis,

\subsubsection{Effect of $Q$ and $R$ Parameters on Model Updating Performance}

For all methods in KF family, the error covariance matrices $Q$ and $R$ governs the accuracy and robustness of the estimation. $Q$ is the process noise covariance for state 


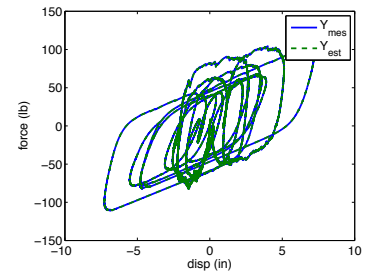

(a) E1-Case $\mathrm{A}$

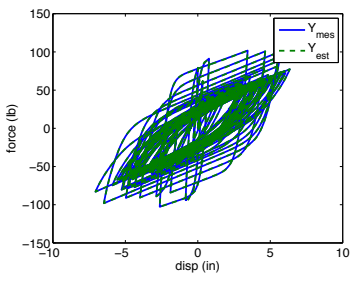

(d) E1-Case B

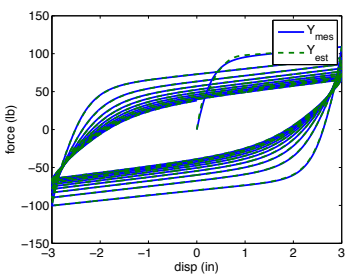

(g) E1-Case C

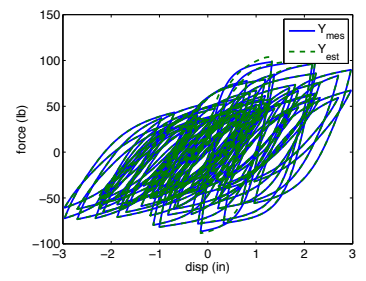

(j) E1-Case D

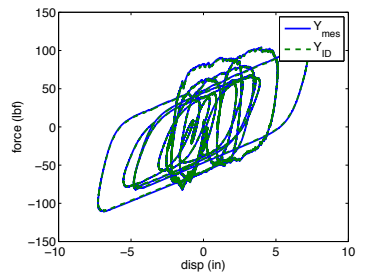

(b) E2-Case A

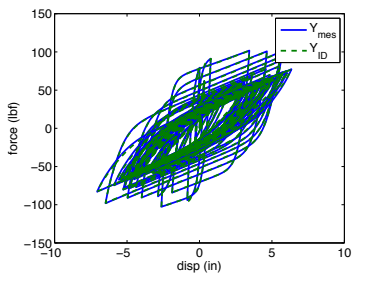

(e) E2-Case B

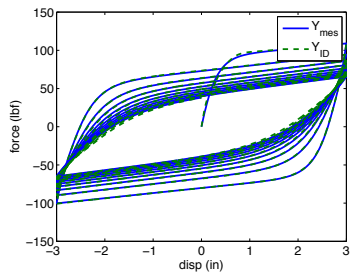

(h) E2-Case C

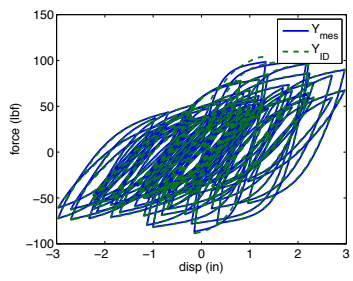

(k) E2-Case D

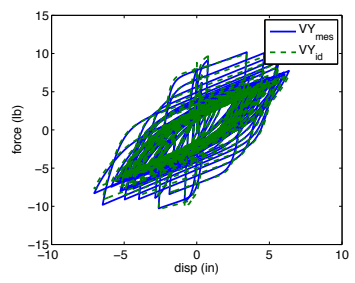

(c) E3-Case A

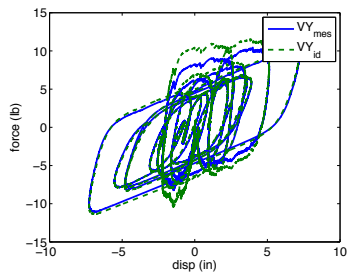

(f) E3-Case B

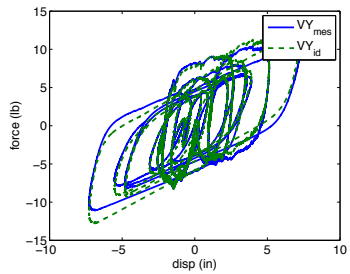

(i) E3-Case C

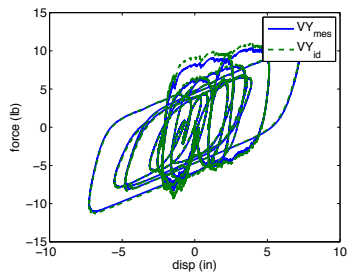

(1) E3-Case D

Figure 8: Real-Time Model Updating Performance and Validation Using Various Excitations, EBW Model 


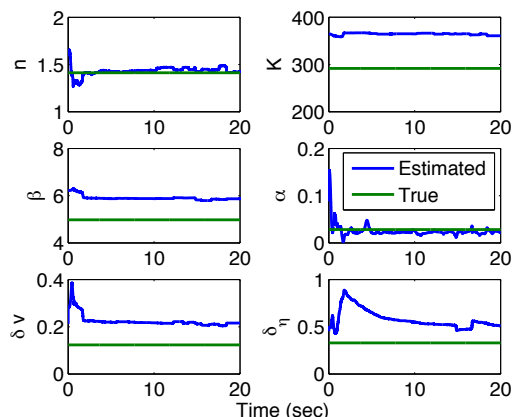

(a) Parameter Convergence - Case A

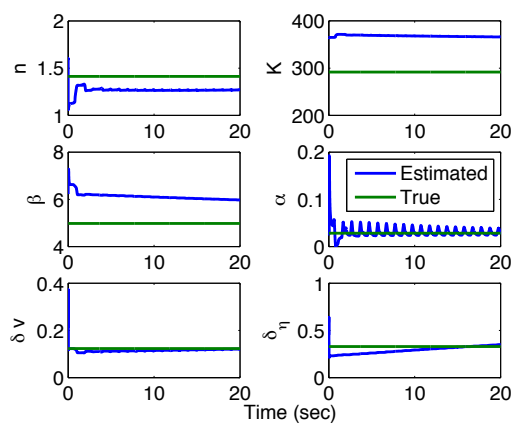

(c) Parameter Convergence - Case C

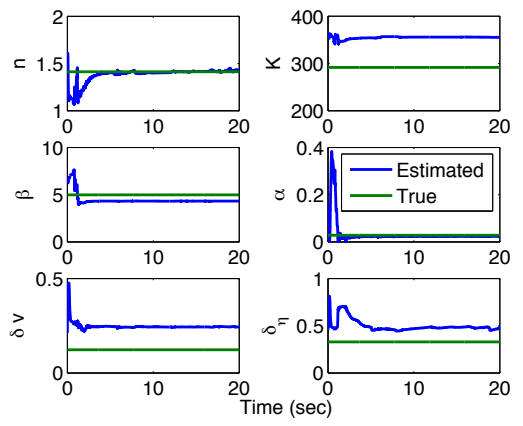

(b) Parameter Convergence - Case B

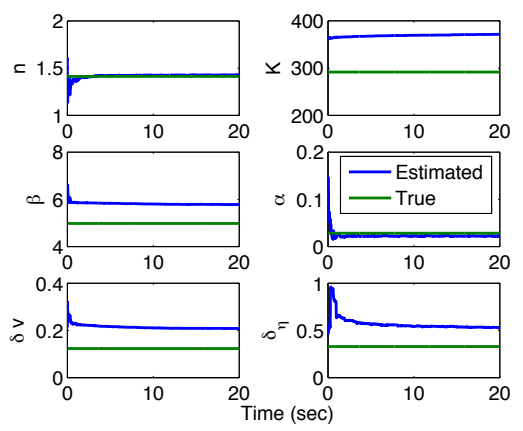

(d) Parameter Convergence - Case D

Figure 9: Parameter Convergence for Excitation Persistence Analysis, Extended BW Model 
estimation, and in the parameter estimation case $Q$ dominates the rate of convergence in identifying the parameters. $R$ is the measurement noise covariance, and in the identification case study $R$ value sets the tolerable error (error threshold) between CUKF output estimation $Y_{\text {est }}$ and the measurement $Y_{\text {mes }}$.

Without loss of generality, for this parametric study the measurement noise covariance is defined as $R=10^{R_{f}}$ and the process noise covariance matrix takes the form of a diagonal matrix as:

$$
Q=10^{Q_{f}} \times\left(\begin{array}{cccc}
\theta_{0}(1) & 0 & \cdots & 0 \\
0 & \theta_{0}(2) & \cdots & 0 \\
\vdots & \vdots & \ddots & \vdots \\
0 & 0 & \cdots & \theta_{0}(L)
\end{array}\right)
$$

To examine the effect of the input on the choice of $Q$ and $R$, two BLWN input cases are considered as indicated in Table 6 and Fig. 10(a). The results are shown in Fig. 10 for the GBW case and demonstrate that the accuracy of model updating (E3) depends strongly on the choice of $Q_{f}$. A smaller $Q_{f}$ yields faster convergence (less variation in each parameter with time), and the steady state value of $Q_{f}$ is reached around $Q_{f}=-7$ for both input cases. Trade-offs in accuracy occur when using larger $R_{f}$ values for all error indices as shown in Fig. 10(c). For small $R_{f}$ values $Q_{f}$ may need tuning, as with $R_{f}=-1$, only a few cases, $Q_{f}=-7,-6,-5$ (solid blue line in Fig 10(b).) run successfully. A similar trend is obtained with case A2 when $R_{f}=1$ (solid red line).

In general, an increase in $R_{f}$ indicates a higher tolerance for estimation error and thus the model updating process tends to be more stable. The results here indicate that there is a co-dependence between $R_{f}$ and $Q_{f}$, and it is worth mentioning that, depending on the test purpose and scenario (level of model uncertainty, convergence speed requirement, input signal and output signal magnitude), the absolute value of $R_{f}$ and $Q_{f}$ should be carefully examined.

\subsubsection{Effect of Initial Guess $\theta_{0}$ Parameter on Model Updating Performance}

In examining the performance of the model updating procedure, another question to address is how the estimation stability and accuracy may be affected by the initial guess 
Table 6: Simulation Cases for Q and R Analysis

\begin{tabular}{c|l|l|l}
\hline Case No. & Signal & $R_{f}$ range & $Q_{f}$ range \\
\hline A1 (GBW) & $0-1 \mathrm{~Hz}$ BLWN & {$[-1: 1: 2]$} & {$[-12: 1:-5]$} \\
A2 (GBW) & $0-3 \mathrm{~Hz}$ BLWN & {$[1: 1: 4]$} & {$[-12: 1:-5]$} \\
\hline
\end{tabular}

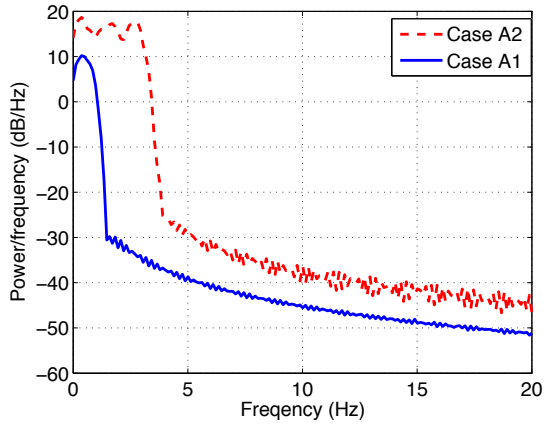

(a) Input Signal PSD - Cases A1 and A2

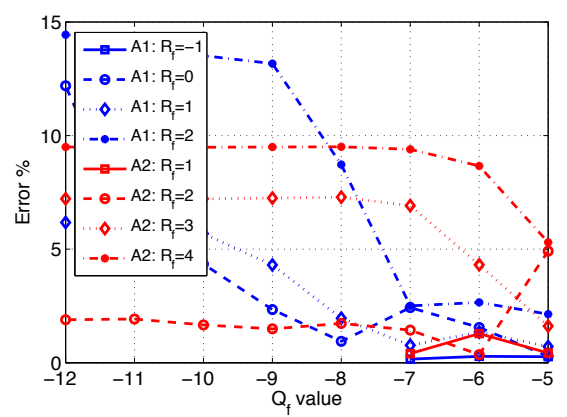

(b) Effect of Q and R on E3 - Case A1 and A2

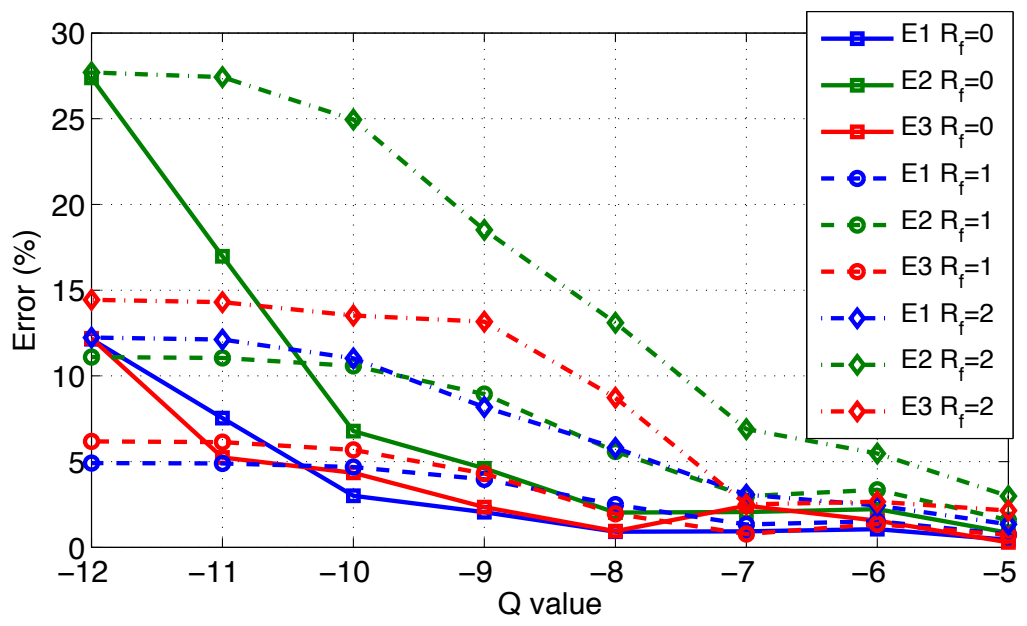

(c) Effect of Q and R on all error indicators - Case A1

Figure 10: Q and R effect on estimation accuracy, General BW model 
(initial condition). To perform this analysis we use an initial guess factor $F_{g}$ which $\theta_{0}$ is defined as $\theta_{0}=F_{g} \cdot \theta_{e}$, where $\theta_{e}$ is the nominal values of the model parameter. $F_{g}$ is chosen from 0.2 to 2.2 , where $F_{g}=0.2$ indicates the initial guess in each parameter is $1 / 5$ of its nominal value and $F_{g}=2.2$ indicates $120 \%$ over estimation in each model parameter.

Five representative models with $F_{g}=[0.2,0.6,1.4,1.8,2.2]$ and the exact model $\left(F_{g}=1.0\right)$ are excited using a sinusoidal input, exhibiting a variety of hysteretic behaviors as demonstrated in Fig. 11(a). The output errors between these erroneous models and the exact models are listed in Table 7. It is clear that the chosen BW initial conditions produce a broad range of hysteretic behaviors and are significant different from the exact model performance.

However, although the initial guesses may be very far away (100\% over estimation) from the exact solution, the CUKF model updating accuracy is not affected greatly 390 as shown in Fig. 11(b). Another variable specific to CUKF algorithm $\lambda_{C}$, which defines the interval constrained unscented transformation (ICUT). From simulation, $\lambda_{C}$ is found to have little influence to model updating performance, thus, is not further discussed.

Table 7: Representative Model Cases

\begin{tabular}{|c|c|}
\hline Case & Error in Response (GBW) \\
\hline$F_{g}=0.2$ & $93.7 \%$ \\
\hline$F_{g}=0.6$ & $52.98 \%$ \\
\hline$F_{g}=1$ & 0 \\
\hline$F_{g}=1.4$ & $42.76 \%$ \\
\hline$F_{g}=1.8$ & $63.94 \%$ \\
\hline$F_{g}=2.2$ & $78.42 \%$ \\
\hline
\end{tabular}




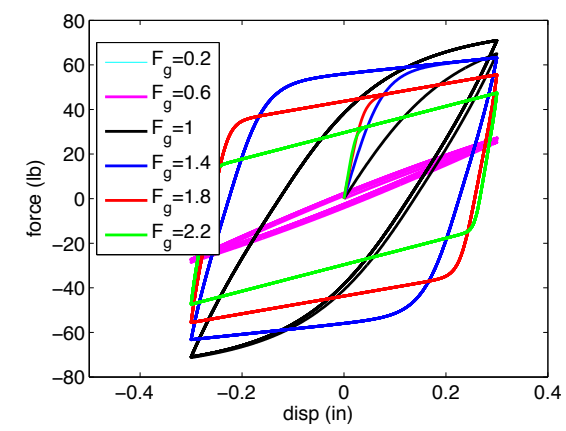

(a) Hysteresis Loop with Different Initial Guess Pa- (b) Estimation Error Indices for Various Initial rameter

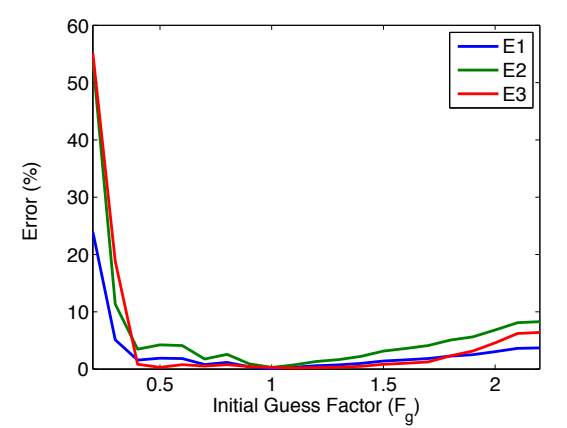

Guesses

Figure 11: Effect of Initial Guess on Estimation Accuracy, General BW model

\section{Conclusion}

RTHSMU performance is analyzed using a numerical example considering a practical case study with three identical energy dissipation plates in a structural system. In this simulation, one of these plates is identified as the experimental substructure in RTHS, although it is simulated here, and the other two instances are in the numerical model. However, with unknown properties of the plates a large error in the global response would result. In simulation, RTHSMU reduces these errors by utilizing identified, real-time model parameters in the target model. The study leads to the following conclusions for implementation of such a model updating procedure.

1) RTHSMU results in improved accuracy in RTHS when the initial target model behavior differs significantly from the true response. If the target model is relatively accurate, it may not always be necessary to implement model updating. Additionally, the initial guess of the target model parameter for CUKF does not significantly affect the stability and convergence of the updating procedure, which indicates that RTHSMU is likely to be successful even with a large initial error in the target model.

2) The accuracy of the model identification procedure depends on the richness of the information (frequency content) in the structural response as this is the input 
to the physical specimen. RTHSMU is not applicable in all situations because reaching the local minimum requires that the system is fully excited. Also, the excitation incompleteness may cause parameter oscillation in identification and not truly converge.

3) The target model complexity should be selected based on the conceptual specimen physical properties. All of the parameters in the model should participate sufficiently in the resulting model behavior. Otherwise, the system identification algorithm is hindered in its ability to estimate the true parameter set, resulting in residual errors.

Other conclusions from this work relate to the CUKF in general. The selection of process noise matrix $Q$ and measurement noise matrix $R$ combination has a large influence on estimation accuracy. Larger $R$ indicates a larger tolerance on error between estimation and measurement, thus the procedure is more robust. Larger $Q$ indicates a slower convergence on estimated parameter, and thus a more accurate solution in general. The combination of $Q$ and $R$ should be carefully studied before implementing CUKF.

\section{Acknowledgments}

This material is based upon work supported in the past by the National Science Foundation under Grant No. CMMI 0927178, CNS 1136075.

The facilities of the George E. Brown Network for Earthquake Engineering Simulation (NEES) Project Warehouse were used for access to data and metadata used in this study (nees.org), funded through CMMI Directorate under Cooperative Agreement Number CMMI-0927178. Open System for Earthquake Engineering Simulation (OpenSees) program was used for FEM simulation is sponsored by the Pacific Earthquake Engineering Research Center.

\section{References}

[1] Aaleti, S., and Sritharan, S., (2009). "A simplified analysis method for characterizing unbonded post-tensioned precast wall systems” Eng Struct, 31(12): 29662975. 
[2] Astrom, K.J., and Wittenmark, B., (2008) "Adaptive Control” Dover Publications.

[3] Baber, T., and Noori, M.N., (1985). "Random vibration of degrading, pinching systems" J. Engrg. Mech., ASCE, 111(8):10101026.

[4] Baber, T., and Wen, Y.K., (1981). "Random vibration of hysteretic degrading systems" J. Engrg. Mech., ASCE, 107(EM6):1069-1089.

[5] Bouc, R., (1971). "Mathematical Model for Hysteresis" Report to the Centre de Recherches Physiques, Marseille, France, pp. 1625

[6] Casciati, F., (1989). "Stochastic dynamics of hysteretic media" Struct. Safety, Amsterdam, 6, 259269.

[7] Chae, Y., Ricles,J.M., and Sause, R., (2014). "Large-scale real-time hybrid simulation of a three-story steel frame building with magneto-rheological dampers", Journal of Earthquake Engineering and Structural Dynamics, 10.1002/eqe.2429.

[8] Christenson, R.E., Lin, Y.Z., Emmons, A.T., and Bass, B., (2008). "Large-Scale Experimental Verification of Semiactive Control through Real-Time Hybrid Simulation”, Journal of Structural Engineering, ASCE, 134(4), pp. 522-535.

[9] Crassidis, J.L., (2005). "Sigma-Point Kalman Filtering for Integrated GPS and Inertial Navigation" AIAA Guidance, Navigation and Control Conference and Exhibit, San Francisco, CA, 2005.

[10] Deierlein, G., Billington, S., Hajjar, J., (2005-2010). Controlled Rocking of SteelFramed Buildings, Network of Earthquake Engineering Simulation, Project 75 (https://nees.org/warehouse/project/75).

[11] Deierlein, G., Krawinkler, H., Ma, X., Eatherton, M., Hajjar, J., Takeuchi, T., Kasai, K., and Midorikawa, M., (2011). "Earthquake resilient steel braced frames with controlled rocking and energy dissipating fuses" Steel Construction 2011 4(3): 171175 .

[12] Eatherton, M., Hajjar, J.F., Ma, X., Pena, A., Deierlein, G.G., Krawinkler, H., and Billington, S., (2008). "Controlled Rocking of Steel Framed Buildings as a 
Sustainable New Technology for Seismic Resistance in Buildings," Creating and Renewing Urban Structures, Chicago, Illinois, September 17-19.

[13] Eatherton, M.R., Hajjar, J.F., Deierlein, G.G., Krawinkler, H., Billington, S., and Ma, X., (2008). "Controlled rocking of steel-framed buildings with replaceable energy-dissipating fuses" Proceedings of the 14th World Conference on Earthquake Engineering, Beijing, China.

[14] Eatherton, M.R., Ma, X., Krawinkler, H., Deierlein, G.G., and Hajjar, J.F., (2014). "Quasi-static cyclic behavior of controlled rocking steel frames" Journal of Structural Engineering, ASCE, 140(11).

[15] Fiorenzani, T., Manes, C., Oriolo, G., and Peliti, P., (2008). "Comparative Study of Unscented Kalman Filter and Extended Kalman Filter for Position/Attitude Estimation in Unmanned Aerial Vehicles" IASI-CNR, R. 08-08, 2008.

[16] Friedman, A., Dyke S.J., Phillips, B.M., Ahn, R., Dong, B., Chae, Y., Castaneda N.,Jiang, Z., Zhang J., Cha, Y., Ozdagli, A., Spencer, B.F., Ricles, J., Christenson, R., Agrawal, A., Sause, R., ’Large-Scale Real-Time Hybrid Simulation for Evaluation of Advanced Damping System Performance" Journal of Structural Engineering, ASCE, to appear.

[17] Garcia-Fernandez, A.F., Morelande, M.R., and Grajal, J.,(2012). "Truncated unscented Kalman filtering" IEEE Trans. Signal Process., 60(7):3372 -3386.

[18] Hashemi, M.J., Masroor,A., and Mosqueda, G., (2014). "Implementation of online model updating in hybrid simulation”, Earthquake Engineering and Structural Dynamics, 134(43), pp. 395-412.

[19] Ikhouane, F. and Rodellar, J., (2007) "Systems with hysteresis: Analysis, identification and control using the Bouc-Wen model", John Wiley \& Sons.

[20] Ismail, M., Ikhouane, F., and Rodellar, J., (2009). "The hysteresis BoucWen model, a survey" Arch Comput Meth E, 262009):161188. 
[21] Julier, S.J., and Uhlmann, J.K., (1997). "A new extension of the Kalman filter to nonlinear systems” Proc. SPIE, 3068(1997):182-193.

[22] Kalman, R.E., (1960). "A New Approach to Linear Filtering and Prediction Problems" Trans. of the ASME - Journal of Basic Engineering, March 1960, pp. 35-45.

[23] Kalman, R.E., and Bucy, R.S., (1961). "New Results in Linear Filtering and Prediction Theory" Trans. of the ASME - Journal of Basic Engineering, 83(1961):95108.

[24] Kwon, O.S. and Kammula, V., (2013). "Model updating method for substructure pseudo-dynamic hybrid simulation", Earthquake Engineering and Structural Dynamics, 134(42), pp. 1971-1984.

[25] Ma, F., Zhang, H., Bockstedte, A., Foliente, G.C., and Paevere, P., (2004). "Parameter Analysis of the Differential Model of Hysteresis" J. Appl. Mech., 71(3):342-349.

[26] Mahin, S.A. and Shing, P.B., (1985). "Pseudodynamic method for seismic testing.” Journal of Structural Engineering, ASCE, 111(7): 1482-1503.

[27] Menegotto, M., and Pinto, P., (1973). "Method of analysis for cyclically loaded RC plane frames including changes in geometry and non-elastic behavior of elements under combined normal force and bending” Proc., Symp. Resistance and Ultimate Deformability of Struct. Acted on by Well-Defined Repeated Loads, IABSE Reports, 13.

[28] Muller, A., (2014). "Real-Time Hybrid Simulation with Online Model Updating", Master Thesis, Western Michigan University, Paper 506.

[29] Nakashima, M., (2001). Development, potential, and limitation of real-time online (pseudo-dynamic) testing , Mathematical, Physical and Engineering Sciences, 359: 1851-1867.

[30] Reinhorn, A.M., Madan, A., Valles, R.E., Reichmann, Y., and Mander, J.B.,(1995). "Modeling of masonry infill panels for structural analysis” Tech. Rep. NCEER-95-0018, State University of New York at Buffalo, Buffalo, N.Y. 

of a Hysteretic Structural System" Journal of Structural Engineering, 140(3), 04013082 . 
[41] Takanashi, M., Udagawa, K., Seki, M., Okada, T., and Tanaka, H., (1975). Nonlinear earthquake response and analysis of structures by a computer-actuator online system, Bulletin of Earthquake Resistant Structure Research Center, University of Tokyo, Tokyo, Japan, Vol 8.

[42] Takanashi, K., and Nakashima, M., (1987). Japanese activities on on-line testing.

[43] Teixeira, B., Torres, L., Aguirre, L., and Bernstein, D., (2010). "On unscented Kalman filtering with state interval constraints" Journal of Process Control, 20(1):45-57.

[44] Vachhani, P., Narasimhan, S., and Rengaswamy, R.,(2006). "Robust and reliable

[48] Wen, Y.K., (1976). "Method of Random Vibration of Hysteretic Systems" Journal of Engineering Mechanics Division, ASCE, 102(EM2):249263.

[49] Wu, B. and Wang, T., (2014) "Model updating with constrained unscented estimation via unscented recursive nonlinear dynamic data reconciliation" J Process Contr, 16(2006):10751086.

[45] Wan, E.A., and Merwe, R.V.D., (2000). "The unscented Kalman filter for nonlinear estimation" Adaptive Systems for Signal Processing, Communications, and Control Symposium 2000, 153158.

[46] Wang, T., Wu, B., (2013). "Real-time Hybrid Testing with Constrained Unscented Kalman Filter" 5th International Conference on Advances in Experimental structural Engineering, Taipei, Taiwan, Nov. 8-9.

[47] Wendel, J., Metzger, J., Moenikes, R., Maier, A., and Trommer, G.F., (2006). "A Performance Comparison of Tightly Coupled GPS/INS Navigation Systems Based on Extended and Sigma Point Kalman Filters" Journal of the Institute of Navigation, 53(1),2006.

Kalman filter for hybrid testing” Smart Structures and Systems, 14(6): 1105-1129. 
[50] Wu, M., and Smyth, A., (2008). "Real-time parameter estimation for degrading and pinching hysteretic models" Int J Non-Linear Mech, 43(9):822-833. 